\title{
Early detection of subjective memory impairment in Parkinson's disease using cerebral perfusion SPECT
}

\author{
In-Uk Song ${ }^{\text {a }}$, Joong-Seok Kim ${ }^{\mathrm{a}}$, Sung-Woo Chung ${ }^{\mathrm{a}}$, Kwang-Soo Lee ${ }^{\mathrm{a}}$, Jin-Kyoung Oh ${ }^{\mathrm{b}}$ and \\ Yong-An Chung,** \\ ${ }^{a}$ Department of Neurology, College of Medicine, The Catholic University of Korea, Seocho-gu, \\ Banpo-dong 505 Seoul, 137-701, Korea \\ ${ }^{b}$ Department of Radiology, College of Medicine, The Catholic University of Korea, Seocho-gu, Banpo- \\ dong 505 Seoul, 137-701, Korea
}

\begin{abstract}
Cognitive dysfunction is a common feature of Parkinson's disease (PD). Recent research has focused on the detection and management of subjective memory impairment (SMI) as the stage that precedes mild cognitive impairment (MCI). Nevertheless, few clinical studies have biomarkers of SMI in PD. Therefore, this study was designed to investigate differences in perfusion brain SPECT between PD with SMI (PD+SMI) and PD without SMI (PD-SMI) to identify a potential prodromal biomarker of progression to dementia in patients with PD. In this study, 30 PD patients with SMI and 24 PD patients without SMI have been recruited. All subjects underwent perfusion brain SPECT and neuropsychological testing. Brain SPECT images were analyzed by using the SPM program and comparing between patients with PD+SMI and PD-SMI. The PD+SMI and PD-SMI groups did not differ in any neuropsychological tests, except for MMSE. Despite a significant difference in MMSE scores, all scores of both groups were in the normal range. Brain SPECT analysis of PD+SMI patients showed hypoperfusion in the frontal and inferior temporal regions, anterior cingulate and thalamus compared with PD-SMI patients. This pilot study investigated the role of decreased brain perfusion SPECT findings in PD+SMI patients compared with PD-SMI patients as a predictive biomarker of pre-dementia as the stage that precedes MCI in PD. Larger, prospective studies are warranted for further investigation of the pathophysiology of neuronal systems during cognitive decline.
\end{abstract}

Keywords: Parkinson's disease, subjective memory impairment, perfusion SPECT

\section{Introduction}

Parkinson's disease (PD) is categorized by progressive degeneration of dopaminergic neurons in the substantia nigra [1]. In PD, changes in the cholinergic, noradrenergic, and serotonergic systems are present in variable degrees, leading to different motor and non-motor symptoms $[1,2]$. Cognitive dysfunction is a common feature of PD and is also one of the most disabling features [1,2]. An earlier

\footnotetext{
${ }^{*}$ Corresponding author: Yong-An Chung, Department of Radiology, Incheon St. Mary's Hospital, The Catholic University of Korea, \#56 Dongsu-ro, Bupyeong-gu, Incheon, 403-720, Korea. Tel.: 82-32-280-5243; Fax: 82-32-280-5244; E-mail: nm@catholic.ac.kr.
} 
clinical indicator of dementia, even before the evolution of mild cognitive impairment (MCI), may be subjective memory impairment (SMI) [3,4]. SMI is defined by subjective reports of memory worsening while maintaining cognitive performance in the normal adjusted range [4]. It is recognized that cognitive changes may develop even in early-stage PD; thus, the definition of MCI has been formulated in PD (PD-MCI). MCI is ascribed to cognitively impaired patients not fulfilling the dementia criteria, originally in Alzheimer's disease (AD). Estimates of the prevalence of PD-MCI vary widely, with a recent estimate of approximately $26 \%$ of non-demented PD patients. Previous studies have also shown that SMI is a risk factor for cognitive decline and have generated preliminary evidence of AD-type pathology in patients with SMI $[3,5,6]$. Therefore, like MCI in the AD prototype, it has been speculated that the presence of SMI in PD patients plays a pivotal role in the progression of dementia.

The primary goals of many neuroimaging studies that have examined biomarkers of the early phase of cognitive decline have been to detect and delay clinical progression [7,8]. Early prevention of clinical progression to dementia, such as PD with dementia (PDD) and AD, is important since it is a progressive and irreversible neurodegenerative disease with no known treatment. Therefore, researchers must perform studies that focus on the detection and management of SMI as the stage that precedes MCI. Nevertheless, there have been few clinical studies of SMI biomarkers in PD until now.

The brain perfusion single- photon emission computed tomography (SPECT) can identify such changes at an early stage and may soon allow visualization of neurotransmission and receptor activity. Furthermore, brain perfusion SPECT is widely available and provides information regarding alterations in regional cerebral blood flow, which reflects cortical function [9]. Brain perfusion SPECT has been used to identify topographic patterns of brain dysfunction in patients with cognitive disorders [10]. Therefore, this study is designed to investigate differences between PD with SMI (PD+SMI) and PD without SMI (PD-SMI) in perfusion brain SPECT by using the SPM program as a potential prodromal biomarker of progression to dementia. An early, accurate diagnosis of prodromal dementia in PD patients may be valuable since it could allow for proper management and prevention of dementia progression.

\section{Materials and methods}

\subsection{Subjects}

This study was approved by the local ethics committee, and written informed consent was obtained from each patient. All subjects were prospectively recruited, and the study was conducted between April 2012 and October 2013. There were 30 patients with PD+SMI and 47 patients with PD-SMI. All subjects were examined in the Dementia Clinic and the Movement Disorder Clinic of Incheon St. Mary's Hospital. Evaluation procedures consist of a detailed medical history, physical and neurological examination and neuropsychological assessment by using the Mini-Mental State Examination (MMSE), the extended version of the Clinical Dementia Scale (CDR) and the sum of the box score of the CDR (SOB). Additional brain magnetic resonance imaging (MRI) and Tc-99 $\mathrm{m}$ hexamethylpropyleneamine-oxime (Tc-99 m HMPAO) perfusion SPECT were performed in all subjects. Patient medical and neurological histories were obtained from the patients, their family members and other caregivers.

All SMI patients were diagnosed according to the definition of a subjective complaint of memory decline among elderly people in the absence of any objective memory disturbance. Therefore, 
although there is no current consensus on standard criteria, for the purposes of this study, SMI was defined as self-reported impairment, accompanied by normal performance on neuropsychological. All PD patients were diagnosed according to the UK Parkinson's Disease Society Brain Bank Clinical Diagnosis criteria. Motor severity of PD was evaluated by using the Hoehn-Yahr staging (H-Y staging) measure. PD patients who were taking medications reported to influence cognition and memory (e.g., anticholinergic agents), who had any clinical signs compatible with atypical parkinsonian disorders, and who fulfilled the DSM-IV criteria for delirium or amnesic and depressive disorders were excluded in this study. In addition, patients with cerebral lesions on MRI or secondary causes of Parkinsonism which may interfere with the safe conduction of the study were also excluded. All statistical analyses were performed using the SPSS software version 18.0 package. The independent T-test was used for the comparison of continuous variables, and Pearson's chi-squared analyses were used for the comparison of categorical variables. Values are expressed as means and standard deviations. Statistical significance was assumed at a false detection rate of less than 5\% (i.e., $p<0.05)$.

\subsection{SPECT imaging}

Scans acquired for all patients were interleaved in time. Images were acquired 40 minutes after intravenous injection of $1110 \mathrm{MBq}$ of Tc-99m HMPAO using a dual-head gamma camera (NM640, GE Healthcare, Milwaukee, WI, USA) equipped with a low-energy, fan-beam collimator. Subjects were in the supine position with eyes open through the scan. The room was dimly lit, and noise was preserved to a minimum.

Image analyses were done with SPM2 software (based on MatLab version 6.0, The MathWorks, Natick, MA, USA). SPECT data were first corrected for attenuation and scatter, and then were transformed into ANALYZE format. Mean pixel intensity across all slices in the imaging volume was computed. Each pixel was then under the threshold of $80 \%$ of its value to remove background noise and partial volume effects at the edge of the brain. Each SPECT scan was then spatially normalized by 12-parameter affine warping and sinc-linear interpolation onto a SPECT template brain from the Montreal Neurological Institute and reformatted to a 16-bit image of $79 \times 95 \times 68$ voxels, each $2 \times 2 \times 2 \mathrm{~mm}$ in size. These images were spatially smoothed with a $16 \mathrm{~mm}$ full-width-at-halfmaximum Gaussian filter. Normalized regional cerebral blood flow ( $\mathrm{rCBF}$ ) values were computed by dividing the $\mathrm{CBF}$ at each voxel by the global $\mathrm{CBF}$ acquired for each individual.

An ANCOVA model was fitted and a " $t$ " statistic image (SPM (t)) was constructed. The covariant effects of MMSE scores on the regional CBF were estimated according to the general linear model. The composite " $\mathrm{t}$ " statistic image PD compared with SMI-PD was under the threshold of $t>1.72$, corresponding to an uncorrected $p<0.05$ in conjunction with a cluster filter of 50 voxels. T-score clusters were projected onto the standard high-resolution T1-weighted MRI images for anatomic localization and visualization.

\section{Results}

The numbers of subjects, mean age, education level, MMSE score, CDR score and SOB score for each group are shown in Table 1. The PD+SMI and PD-SMI groups did not show any statistically significant differences in the mean duration of $\mathrm{PD}$, daily dosage of levodopa or H-Y stage. On the other hand, there is a significant difference in MMSE score but no difference in CDR, including SOB, 
Table 1

Demographic data and cognitive functions in the pAD and PDD groups and normal controls

\begin{tabular}{llll}
\hline Variables & PD+SMI & PD-SMI & P-value \\
\hline \hline Subjects & 30 & 24 & \\
Men & 13 & 11 & 0.736 \\
Mean age, years & $64.17 \pm 10.14$ & $65.96 \pm 10.99$ & 0.541 \\
Mean education, years & $11.87 \pm 3.56$ & $11.21 \pm 5.85$ & 0.612 \\
Mean duration of PD, months & $36.13 \pm 30.22$ & $28.63 \pm 21.62$ & 0.293 \\
H-Y stage & $1.67 \pm 0.51$ & $1.73 \pm 0.68$ & 0.71 \\
Daily dosage of levodopa(mg) & $340.58 \pm 162.86$ & $285.42 \pm 235.98$ & 0.315 \\
MMSE score & $28.36 \pm 1.03$ & $29.63 \pm 0.58$ & $<0.001$ \\
CDR score & $0.2 \pm 0.24$ & $0.13 \pm 0.22$ & 0.253 \\
SOB score & $0.35 \pm 0.60$ & $0.16 \pm 0.32$ & 0.159 \\
\hline
\end{tabular}

Values are mean \pm standard

deviation

PD: Parkinson's disease, SMI: subjective memory impairment, PD+SMI: PD with SMI, PD-SMI: PD without SMI,

MMSE: Mini- mental state examination, CDR: Clinical dementia rating, SOB: Sum of box of CDR

between these two groups. However, the mean MMSE score of the PD+SMI group was within the normal limit.

Regarding the brain perfusion SPECT analysis of PD+SMI and PD-SMI patients, the PD-SMI group demonstrated more significant hypoperfusion in the anterior cingulate, thalamus, medial frontal regions and inferior temporal regions (Figure 1).

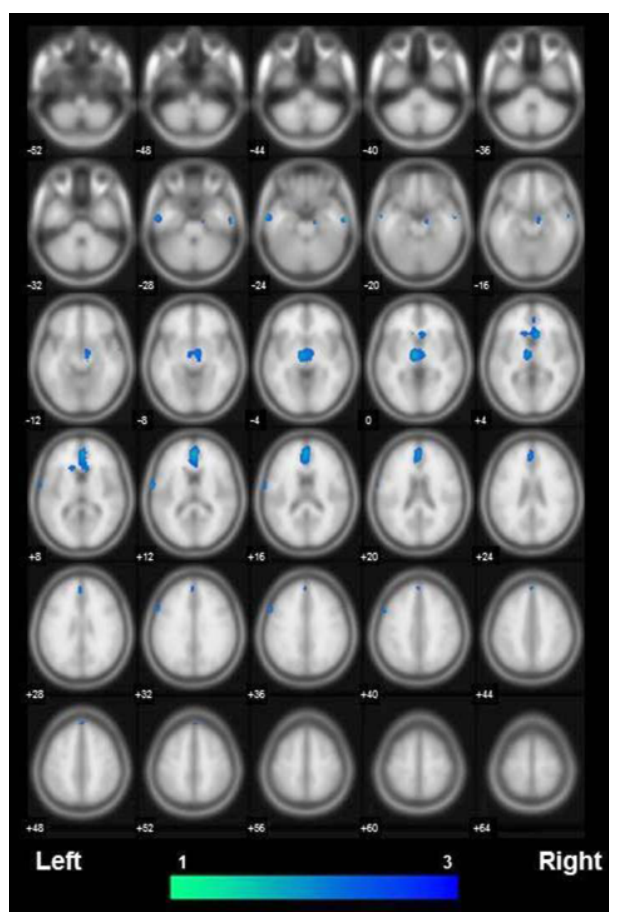

Fig. 1. Group differences in patients with PD+SMI compared with PD-SMI by Fusion of SPM2 results to trans-axial MR images, which were provided as a template. The patients with PD+SMI showed decreased perfusion in medial frontal and inferior temporal regions, anterior cingulate and thalamus compared to patients with PD-SMI. 


\section{Discussion}

The lack of standardized diagnostic tests for neurodegenerative diseases such as PD and dementia greatly limits the potential for an accurate diagnosis and early detection even more so. As a pre-MCI condition, SMI may be helpful in identifying, and therefore treating at-risk individuals before irreversible neuronal loss occurs.

To date, there has been little clinical or neuroimaging research on SMI in PD. Nobili et al. reported that patients with SMI showed no significant differences in brain SPECT compared with healthy subjects [10]. However, another FDG-PET study suggested that patients with SMI have significant cerebral glucose metabolism reductions in the parahippocampal gyrus, parieto-temporal and inferior frontal cortex, fusiform gyrus, and thalamus [11]. A recent neuroimaging study showed significantly decreased gray matter density in the angular gyrus, both medial frontal gyri and anterior cingulate regions in PD+SMI patients compared with PD-SMI patients [12]. The brain SPECT analysis of PD+SMI patients in this study also showed a decreased perfusion pattern in the thalamus, anterior cingulate, medial frontal and inferior temporal regions compared with PD-SMI patients, which is consistent with prior neuroimaging studies [11,12]. In particular, it is well known that the inferior temporal region influences semantic memory, and the anterior cingulate are involved in attention and rational cognitive functions, such as decision-making, empathy, impulse control, and emotion $[12,13]$. Furthermore, executive cognitive function takes place in the frontal lobe. Therefore, it could be speculated via the data that PD+SMI may be an early manifestation of pre-dementia in PD that occurs before MCI.

One limitation of this study is that the diagnosis of SMI has not yet been validated, and a consensus definition has not been reached. Thus, SMI may precede future cognitive decline or represent a predementia state. However, most studies are in agreement with the above-mentioned designation of SMI, and these diagnostic criteria were applied in the present study. It was attempted to address these confounders via neuropsychological tests and neurological examinations performed by an experienced neurologist.

In conclusion, this is the first study to assess functional neuroimaging using perfusion SPECT for patients with PD+SMI and PD-SMI. This pilot study evaluated the role of decreased brain perfusion SPECT findings in PD+SMI patients compared with PD-SMI patients as a predictive biomarker of pre-dementia as the stage that precedes MCI in PD. Prospective and clinicopathologic studies which are aimed at investigating the pathophysiology of neuronal systems during cognitive decline from PD+SMI to PDD are needed. Ideally, these studies will also identify whether progression coincides with the aforementioned radiologic observations and whether this parallels objective cognitive decline.

\section{References}

[1] I.U. Song, J.S. Kim, I.S. Park, Y.D. Kim, H.J. Cho, S.W. Chung et al., Clinical significance of homocysteine (hcy) on dementia in Parkinson's disease (PD), Arch. Gerontol. Geriatr. 57 (2013), 288-291.

[2] A.A. Kehagia, R.A. Barker and T.W. Robbins, Neuropsychological and clinical heterogeneity of cognitive impairment and dementia in patients with Parkinson's disease, Lancet. Neurol. 9 (2010), 1200-1213.

[3] L. Scheef, A. Spottke, M. Daerr, A. Joe, N. Striepens, H. Kolsch et al., Glucose metabolism, gray matter structure, and memory decline in subjective memory impairment, Neurology 79 (2012), 1332-1339.

[4] R. Stewart, Subjective cognitive impairment, Curr. Opin. Psychiatry 25 (2012), 445-450.

[5] B. Reisberg, M.B. Shulman, C. Torossian, L. Leng and W. Zhu, Outcome over seven years of healthy adults with and without subjective cognitive impairment, Alzheimer's Dement 6 (2010), 11-24. 
[6] F. Jessen, B. Wiese, C. Bachmann, S. Eifflaender-Gorfer, F. Haller, H. Kolsch et al., Prediction of dementia by subjective memory impairment: Effects of severity and temporal association with cognitive impairment, Arch. Gen. Psychiatry 67 (2010), 414-422.

[7] H.S. Kim, M.H. Choi, H.J. Yoon, H.J. Kim, U.H. Jeoung, S.J. Park et al., Cerebral activation and lateralization due to the cognition of a various driving speed difference: An fMRI study, Biomed. Mater. Eng. 24 (2014), 1133-1139.

[8] T. Liu, P. Lin, Y. Chen and J. Wang, Electroencephalogram synchronization analysis for attention deficit hyperactivity disorder children, Biomed. Mater. Eng. 24 (2014), 1035-1039.

[9] A. Paschali, L. Messinis, E. Lyros, C. Constantoyannis, Z. Kefalopoulou, V. Lakiotis et al., Neuropsychological functions and rCBF SPECT in parkinson's disease patients considered candidates for deep brain stimulation, Eur. J. Nucl. Med. Mol. Imaging 36 (2009), 1851-1858.

[10] F. Nobili, F. De Carli, G.B. Frisoni, F. Portet, F. Verhey, G. Rodriguez et al., SPECT predictors of cognitive decline and alzheimer's disease in mild cognitive impairment, J. Alzheimer's Dis. 17 (2009), 761-772.

[11] L. Mosconi, S. De Santi, M. Brys, W.H. Tsui, E. Pirraglia, L. Glodzik-Sobanska et al., Hypometabolism and altered cerebrospinal fluid markers in normal apolipoprotein E E4 carriers with subjective memory complaints, Biol. Psychiatry 63 (2008), 609-618.

[12] J.Y. Hong, J.E. Lee, Y.H. Sohn and P.H. Lee, Neurocognitive and atrophic patterns in Parkinson's disease based on subjective memory complaints, J. Neurol. 259 (2012), 1706-1712.

[13] J.M. Allman, A. Hakeem, J.M. Erwin, E. Nimchinsky and P. Hof, The anterior cingulate cortex, the evolution of an interface between emotion and cognition, Ann. N. Y. Acad. Sci. 935 (2001), 107-117. 